Other slide sets available from the A.S.P. include:

1. The Moon Kit (18 slides)

2. Mars Kit (6 slides)

3. Venus Kit ( 6 slides)

4. The Solar System Close-Up (2 sets of 50 slides, 1 set of 30 slides, with captions by David Morrison)

5. Voyager at Uranus (15 slides)

6. Halley's Comet Revealed (17 slides, with a booklet by John C. Brandt)

7. Splendors of the Universe (15 slides with captions and background information by David Malin; shows color views of Southern Hemisphere objects)

8. The Sky at Many Wavelengths (11 slides, with an extensive booklet by Christine Jones and William Forman)

9. The Infrared Universe: An IRAS Gallery (25 slides with captions and background information by Charles Beichman)

10. The Search for Extraterrestrial Intelligence (20 slides with captions and a basic introduction by Frank Drake)

11. Portraits of the Solar System (20 slides of paintings by William Hartmann)

12. Supernova 1987A (6 slides)

13. Science from the Space Shuttle (30 slides with captions by Michael Lampton)

We welcome suggestions for other sets we should do and also offers to put together a set for us on a subject of special interest to you.

For a complete catalog of A.S.P. slide sets, please write to: Catalog Requests Dept., Astronomical Society of the Pacific, 390 Ashton Ave., San Francisco, CA 94112, U.S.A.

\title{
DYNAMIC HUMAN (ASTRONOMICAL) MODELS
}

\section{Jeanne E. Bishop}

Director, Westlake Schools Planetarium, 24525 Hilliard Road, Westlake, Ohio 44145, U.S.A.

Many educators have found that models can add a lot to the understanding of astronomical concepts. Attractive commercial models of the solar system and celestial globes are readily available. Many models can be constructed of common materials - I found in my doctoral dissertation work that models made and ma- 
nipulated by students increased retention of concepts of the celestial sphere and rotation, the seasons, lunar phases, and planet motions. ${ }^{1}$

But there is another type of model which I have found very successful. I call it the "dynamic human model." Actually, the concept is not new. I know that many good teachers have a favorite demonstration in which one or more students represent the Earth, sun, or moon. Also, the Elementary-School Science Project of the University of Illinois contained activities for student models to distinguish between Ptolemaic and Copernican systems. ${ }^{2}$ However, I do not think the idea has been extended as far as it should be. I would like to suggest ideas for dynamic human models beyond what I have seen elsewhere.

Before describing examples, let me indicate good reasons for utilizing the technique. First, students enjoy a model in which they both participate and cooperate with others, and this appears to be true for elementary school through university. A recent review of research studies involving student-student interaction concludes that a group project requiring interdependence is likely to help everyone in the group master the concepts studied. Also it appears that retention is enhanced in the cooperative setting. ${ }^{3}$

There are other reasons. The spatial visualization required in many astronomical concepts requires time... The more complex the spatial array, the more time is needed. Dynamic human models take time to explain, arrange, and then enact. Spatial visualization is sometimes difficult - frequently girls have more trouble than boys, and the more detailed concrete three-dimensional models incorporated in astronomy studies, the better learning will be. Models that show motion or changes are superior to those that do not, and mechanical models are usually expensive, difficult, or impossible to construct.

As I developed ideas for dynamic human models, I learned that several conditions (at least) are necessary for success. First, students must be serious and cooperative so that they become quiet at appropriate moments and assume the required roles. It is important to sit down prior to the activity and discuss what is to be done, the value of the activity, and the roles to be taken. Not only do students become sympathetic to the purpose of the activity, but they frequently make suggestions or ask questions that lead to modifications that will improve the model. With everyone united in purpose, the model probably will be a success.

Second, the concept and degree of detail should match the ability and previous knowledge of the group of students. It would not be effective to have a group of primary students (6-8 years old) model intricate details of star evolution or the electromagnetic spectrum. I have found, however, that very young capable children in gifted and talented programs can learn advanced concepts via this method of dynamic models better than via any other procedure.

Third, a fairly large area is needed for the dynamic human models. A gym, a lecture room without chairs, and outdoor areas work well. Particularly if you would like to provide some semblance of scale, the large spaces are required.

Today I would like to demonstrate two of my ideas on dynamic human models appropriate for upper elementary students, secondary students, and university stu- 
dents. The first model is for dynamic equilibrium in a stable star and the evolution of that star. The second model is for different wavelengths of light.

\section{Model: Dynamic Equilibrium and Star Evolution}

Two groups of students (two halves of a class) are identified. One group becomes inner gas parts of a giant star, who hold up hands toward the outside, exerting radiation pressure. The second group becomes outer gas parts of the giant star, who hold up hands toward the inside. The inner group pushes out against the hands of the outer group, who push inward. The star is in equilibrium, and it neither expands or shrinks. On cue, the dynamic equilibrium is upset, and inner forces push the outer back quickly and dramatically. This models a supernova explosion. One inner person remains spinning rapidly (conserving angular momentum), assuming the role of a black hole/neutron star. The model can be greatly enriched with a) a flashlight for the neutron star, making a pulsar, b) different "winds" or envelopes leaving the outside in stages, as shown happened in Supernova 1987A, and c) some exploded material initiating new star formation and/or becoming incorporated into a next-generation star.

\section{Model: Wavelengths of Light}

Mark parallel lines on the floor or ground. Different numbers can be used, although three works well. The three lines represent paths of red, yellow, and blue light. Place sticky dots of different colors on the lines. The red light line has red dots, the yellow light line has yellow dots, and the blue light line has blue dots. Place the red dots about $1.0 \mathrm{~m}$ apart, the yellow dots about $0.8 \mathrm{~m}$ apart, and the blue dots about $0.6 \mathrm{~m}$ apart. One student walks along each line simulating a traveling transverse light wave. All students begin by crouching on the first dot of a line. On reaching the next dot in a line, the student is to be fully upright. On each subsequent dot the student is crouched or upright, alternately. All students should progress down the light lines at the same rates, illustrating that all light waves travel at the same velocity in the same medium. To accomplish this, students moving on the lines with closer-spaced dots will have to go up and down faster. This demonstrates that yellow and blue light have more energy than red light, and have higher frequencies. Waves in non-visible sections of the electromagnetic spectrum also can be modeled. the scattering of short waves and the ability to see red light on the other side of clouds can be shown.

\section{Other Ideas for Dynamic Human Models}

- Eclipses, in which students assume roles of the Earth, the sun, and the moon.

- Binary-star system, in which two students revolve about a common center of gravity. The "stars" can form an eclipsing binary if the head of one student partly or totally blocks the head of the other from a distant student's perspective. 
- Different types of galaxies.

- Interacting galaxies, in which groups of students pass with little or no bumping. Some students in roles as "gas clouds" are pulled away in the direction of the galaxy, as in M51. (Galaxies do not need to be the same size.)

- The sun and planets. "Planets" closer to the sun have greater velocities. To show how Neptune can be closer to the sun than Pluto, mark (with chalk, string, or tape) a long eccentric orbit for Pluto which is closer to the "sun" than Neptune in one section. "Earth" can note sidereal and synodic periods of the planets.

- The Oort Cloud, developing comets. A comet "tail" can be a prop of a long piece of aluminum foil, unrolled as the "comet" approaches the "sun." The "comet" walks swiftly about the sun at perihelion, trying to keep its tail pointed away from the sun. "Comets" can be periodic or not.

- Formation and evolution of the sun.

- Comparison of evolution of a sun-sized star and a supergiant, showing different late-life stages and the much longer life of the smaller star.

- The big bang. This can be simple or quite complex. In a complex model, students can model the formation of different types of matter and the separation of forces.

- Adiabatic and isothermal scenarios of galaxy formation.

- Structure of the sun.

- Structure of the center of the Milky Way Galaxy.

- Proton-proton and the CNO cycles, in which students assume roles as different nuclides, protons, neutrinos, and energy.

- Chemical species and structures: giant molecular clouds, nucleus of Comet Halley, including the clathrate hydrate structure, composition of Earth's early atmosphere and its evolution.

- Lunar Phases.

- Latitudinal and longitudinal lunar librations.

- Seasons.

- Sidereal and synodic days compared.

- Sidereal and synodic months compared.

Two final comments about using this teaching procedure.... First, I have found that it is helpful to have labels students can wear, when the model is complex. With many different roles, the labels help everyone comprehend what is happening. Labels can be signs with string or yarn worn about the neck, or pin-on cards. Sometimes props in the form of spheres held by the students or colors they are wearing will enhance the model.

Second, I believe it is important to explain what is wrong with any model, as 
well as what is right about it. Student misconceptions can be minimized in this way. For example, in our dynamic equilibrium model, the process is not present only at one radius from the center but at every point within the star. In our wavelenghts model, the waves actually travel much more rapidly than students can move, and unless polarized, vibration is in many planes centered along a line.

I would appreciate hearing from others who have tried dynamic models and who may now create come new ones.

\section{References}

${ }^{1}$ Bishop, Jeanne E. The Developing and Testing of a Participatory Planetarium Unit Emphasizing Projective Astronomy Concepts and Utilizing the Karplus Learning Cycle, Student Model Manipulation, and Student Drawing with Eighth Grade Students. Unpublished doctoral dissertation, University of Akron, Ohio, U.S.A., 1980.

${ }^{2}$ Astronomy: The Universe in Motion (Book 2). Elementary-School Science Project, University of Illinois, Urbana, U.S.A., 1963.

${ }^{3}$ Johnson, Roger T. and Johnson, David W. "Encouraging Student/Student Interaction." National Association for Research in Science Teaching News. U.S.A. December, 1986.

\section{Discussion}

R.W. Clark: You have stated that females have more difficulty with spatial concepts than males. Is this statement based on any factual reserach, or on some assumptions?

J.E. Bishop: There are many studies that have found that there is both a difference in spatial ability between males and females and a difference in learning material involving spatial ability between males and females. Some of these, including my own doctoral research, have found this for astronomy concepts, the effect is not strong at young ages, but develops through adolesence. Why this happens is not adequately explained. Many feel it is related to environmental treatment. 